\title{
NUMERICAL INVESTIGATION OF THE FLOW PAST A CAVITY
}

\author{
Tim Colonius*, Amit J. Basu ${ }^{\dagger}$, and Clarence W. Rowley ${ }^{\ddagger}$ \\ Division of Engineering and Applied Science \\ California Institute of Technology \\ Pasadena, CA 91125
}

\begin{abstract}
Numerical simulations are used to investigate the resonant instabilities in the flow past an open cavity. The compressible Navier-Stokes equations are solved directly (no turbulence model) for two-dimensional cavities with laminar boundary layers upstream. The computational domain is large enough to directly resolve a portion of the radiated acoustic field. The results show a transition from a shear layer mode, for shorter cavities and lower Mach numbers, to a wake mode for longer cavities and higher Mach numbers. The shear layer mode is well characterized by Rossiter modes. The wake mode is characterized instead by a large-scale vortex shedding with Strouhal number independent of the Mach number. The vortex shedding causes the boundary layer to periodically separate upstream of the cavity. The wake mode oscillation is similar to that reported by Gharib and Roshko (J. Fluid Mech.,177, 1987) for incompressible flow with a laminar upstream boundary layer. The results suggest that laminar separation upstream of the cavity edge is the cause of the transition to wake mode.
\end{abstract}

\section{Introduction}

Recently, the possibility of using flow control to reduce resonant oscillations in subsonic and transonic flows over open cavities has attracted much attention. The (open-loop) introduction of flow disturbances has been studied both experimentally and numerically $\left(e . g .^{1-5}\right)$. Significant reductions in sound pressure levels (around $20 \mathrm{~dB}$ ) have been achieved through upstream mass injection ${ }^{4}$ as well as piezoelectric flaps. ${ }^{5}$ Closed-loop feedback control has also been studied, ${ }^{5-7}$ and promises similar reductions in noise, but with much lower power input. ${ }^{5}$ It should be noted that it has long been known that passive devices, such as spoilers and ramps, can attenuate cavity oscillations under certain conditions.

\footnotetext{
* Assistant Professor, Member AIAA

$\dagger$ Visiting Associate, Member AIAA (on leave from JNCASR, Bangalore)

$\ddagger$ Graduate Research Assistant

Copyright (C) 1999 by the authors. Published by the American Institute of Aeronautics and Astronautics, Inc. with permission.
}

In compressible flows, cavity resonance is thought to arise from a feedback loop involving (i) shear layer instability and the growth of vortices in the shear layer, (ii) the impingement of the vortices at the downstream edge, and subsequent scattering of acoustic waves, (iii) the transmission of acoustic waves upstream, and (iv) their conversion to vortical fluctuations at the cavity leading edge (receptivity). The first description of this feedback process is credited to Rossiter. ${ }^{8}$ His semi-empirical formula to predict the measured resonant frequencies remains widely used. Analytical models to predict the frequency have been developed, ${ }^{9}$ and semi-empirical models that predict the amplitudes are also available. ${ }^{10}$

While existing models are effective as general design tools, there is as yet no way, for a given set of conditions, to determine the dominant mode of oscillation, the amplitude of oscillation, and, especially, nonlinear interactions between modes. Feedback controllers can be designed without detailed flow models (e.g. adaptive control), but a better understanding of the flow physics will lead to more effective and efficient control. Recent experiments ${ }^{11}$ have underscored the complicated nonlinear interaction of the different modes, and the possibility of mode-switching. Fabris and Williams ${ }^{12}$ have also recently investigated these issues for low Mach number cavities.

Direct Numerical Simulations (DNS) provide a means to study the details of the modes of oscillation and their interactions, albeit at low Reynolds number. In this paper we present a high-order-accurate method for solving the compressible Navier-Stokes equations in the cavity geometry and give results for two-dimensional computations. The generated acoustic field is directly resolved in the computation, and the domain is made large enough to include a portion of the radiated acoustic field. Investigations of three-dimensional effects, and the introduction of feedback control in the computations will be presented in future publications. Previous numerical studies of compressible cavity flows have used the two-dimensional unsteady RANS (Reynolds' Averaged Navier-Stokes) equations with a $k-\epsilon$ turbulence model. ${ }^{3,13,14}$ The effectiveness of compressible turbulence models on separated oscillating flows, and 
especially their radiated acoustic field (which, as noted above, is an integral part of the resonant instability modes) remains an open question. It needs to be stressed that in the context of two-dimensional flows, we use the term "direct" simulation to imply that there is no turbulence model. In this case the flow is an unstable laminar flow that is confined to evolve in only two-dimensions. The turbulent cavity flow is of course three-dimensional, but it is thought that in many cases the resonant modes are approximately two-dimensional.

\section{$2 \quad$ Numerical Method}

The numerical method used here is very similar to methods used previously for direct computations of sound generation in mixing layers and jets and other canonical problems,${ }^{15-18}$ wherein the fully compressible Navier-Stokes equations are solved. These studies have shown the efficacy of sixth-orderaccurate compact finite-difference schemes ${ }^{19}$ in resolving acoustic fields with velocity fluctuations five orders of magnitude smaller than near field fluctuations. ${ }^{15}$ Time integration is performed with a fourth-order Runge-Kutta method. This combination of schemes results in very low numerical dissipation, which allows accurate wave propagation, and the method relies on physical viscosity for stability.

Boundary conditions play a key role in aeroacoustic computations. Artificial boundaries (inflow/outflow/normal) must allow vortical and acoustic waves to pass freely, with minimal reflection. It is important to distinguish between two types of reflections that may occur: "smooth" reflections, which arise due to approximations in the continuous boundary condition formulation, and "spurious" or saw-tooth reflections, which arise due to the dispersive nature of finite difference schemes. ${ }^{20}$ For equations of motion linearized about a uniform mean flow, it is possible to derive boundary conditions that are nonreflecting for both types of waves, to arbitrarily high order of accuracy. ${ }^{20}$ For nonlinear equations, especially at outflow boundaries, the interaction of disturbance amplitudes and mean flow gradients severely limits the accuracy of such boundary conditions developed for linearized problems. Several treatments that rely on a "buffer" zone near the computational boundary have been suggested to remedy this situation. These include combinations of grid stretching and filtering, ${ }^{21}$ and the addition of artificial convection velocities and damping terms to the equations $\left(e . g .^{22}\right)$. These "buffer" conditions are usually combined with low-order accurate, but robust, nonreflecting boundary conditions, such as one-dimensional characteristic wave decom- positions. $^{23,24}$

Figure 1 shows a schematic diagram of the computational domain. A Cartesian grid is used, with clustering of nodes near all the walls. Analytical hyperbolic tangent mappings are used for the grid stretching. The code is parallelized using a domain decomposition method. Typical grids (see caption of figure 2) contain about half a million grid points. The code has been run on 8 to 32 processors of an IBM SP2. The wall is assumed to be isothermal at the same temperature as the freestream (therefore transport properties are assumed constant, and the Prandtl number is taken as 0.7). For the inflow, outflow and normal boundaries, the one-dimensional boundary conditions of Poinsot and Lele ${ }^{24}$ are used, together with artificial damping terms in a buffer region. ${ }^{22}$ These terms, of the form $\sigma\left(\mathbf{q}-\mathbf{q}_{\text {target }}\right)$ are added to the right-hand sides of the equations in conservative form ( $\mathbf{q}$ is a vector of the conservative dependent variables). The damping, $\sigma$, varies smoothly from a constant value at the boundary to zero at the edge of the buffer. For the isothermal wall boundary conditions, including the cavity edges, the formulation recommended by Poinsot and Lele is also used.

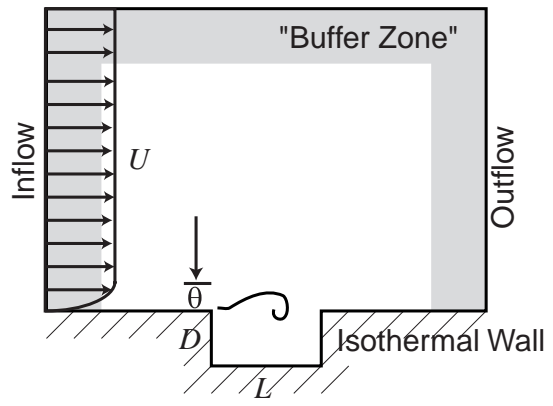

Figure 1: Schematic diagram of cavity configuration and computational domain.

The simulations are initiated by spanning the cavity with a Blasius flat-plate boundary layer. The equations are nondimensionalized with the cavity depth, ambient density, and freestream velocity, $U$. The following parameters may be independently varied: the length of the cavity relative to the initial boundary layer thickness at the cavity leading edge, $L / \theta$; the Reynolds number, $\operatorname{Re}_{\theta}$, based $U, \theta$, and the kinematic viscosity in the ambient flow, $\nu$; the Mach number of the freestream, $M$; and the cavity length to depth ratio, $L / D$. Because of the expense, only a relatively small portion of parameter space may be investigated. In the present paper, we concentrate on two-dimensional computations with 


\begin{tabular}{|l|ccccl|}
\hline Run & $L / D$ & $L / \theta$ & $M$ & $\mathrm{Re} \theta$ & Mode $\left(C_{D}\right)$ \\
\hline L1 & 1 & 20.3 & 0.6 & 73.9 & $\mathrm{~S}(0.002)$ \\
L2 & 2 & 52.8 & 0.6 & 56.8 & $\mathrm{SL}(0.008)$ \\
L3 & 3 & 75.0 & 0.6 & 60.0 & $\mathrm{M}(0.031)$ \\
L4 & 4 & 102.1 & 0.6 & 58.8 & $\mathrm{~W}(0.227)$ \\
L5 & 5 & 123.3 & 0.6 & 60.8 & $\mathrm{~W}(0.404)$ \\
\hline 4M2 & 4 & 102 & 0.2 & 58.8 & $\mathrm{SL}$ \\
4M3 & 4 & 102 & 0.3 & 58.8 & $\mathrm{M}$ \\
4M4 & 4 & 102 & 0.4 & 58.8 & $\mathrm{~W}$ \\
4M5 & 4 & 102 & 0.5 & 58.8 & $\mathrm{~W}$ \\
4M6 & 4 & 102 & 0.6 & 58.8 & $\mathrm{~W}$ \\
4M7 & 4 & 102 & 0.7 & 58.8 & $\mathrm{~W}$ \\
4M8 & 4 & 102 & 0.8 & 58.8 & $\mathrm{~W}$ \\
\hline 2M2 & 2 & 52.8 & 0.2 & 56.8 & $\mathrm{~S}$ \\
2M3 & 2 & 52.8 & 0.3 & 56.8 & $\mathrm{~S}$ \\
2M4 & 2 & 52.8 & 0.4 & 56.8 & $\mathrm{SL}$ \\
2M5 & 2 & 52.8 & 0.5 & 56.8 & $\mathrm{SL}$ \\
2M6 & 2 & 52.8 & 0.6 & 56.8 & $\mathrm{SL}$ \\
2M7 & 2 & 52.8 & 0.7 & 56.8 & $\mathrm{SL}$ \\
2M8 & 2 & 52.8 & 0.8 & 56.8 & SL \\
\hline
\end{tabular}

Table 1: Run parameters. Abbreviations for modes are: $\mathrm{S}=$ Steady, $\mathrm{SL}=$ Shear Layer, $\mathrm{W}=$ Wake, $\mathrm{M}$ $=$ Mixed.

laminar upstream boundary layers. Table 1 shows relevant parameters for the runs performed.

For the present flow, and for resonant flows in general, it is of critical importance that instabilities are independent of the location of the boundaries, and the boundary treatment. This is because repeated spurious reflections of waves can lead to self-forcing of the flow, in a process indistinguishable from the physical instability $\left(e . g \cdot{ }^{25}\right)$. We have run several cases with variable boundary location and grid spacing, in order to find appropriate boundary locations, as well as grid convergence. The results of one such test are shown in figure 2. Plotted is the normal velocity at a single point, $y=0$ and $x=3.13 D$, with the same parameters as run L4. Other probe locations yielded similar results. Note that time is normalized by the freestream velocity and the total length of the computational domain for the reference case. Over 3 to 4 flow-through times, the results are nearly identical, independent of grid resolution and boundary location. Small differences are apparent at later times, which is not unexpected given the chaotic nature of the flow. We conclude that the location of the boundaries and grid resolution for run L4 (see the figure caption) are sufficient, and similar locations and resolutions were used in the other runs in table 1 .

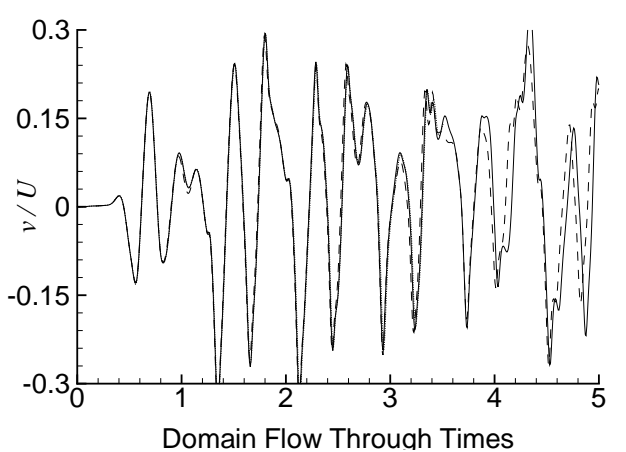

Figure 2: Effect of boundary position and grid resolution on the normal velocity at $y=1.57 D$ and $x=3.13 D$. Reference case (-) has downstream boundary: $10.6 D$, upstream: $-4.3 D$, normal: $9.2 D$. Grid has $1152 \times 384$ points above the cavity in the streamwise and spanwise directions, respectively, and $384 \times 94$ points in the cavity. Finer grid case $(----)$ has same boundaries as run L4, but $50 \%$ more grid points (in each direction). Larger domain case (.....) extends to $15 D$ downstream and $15 D$ in the normal direction. Note that the dotted line falls nearly directly on top of the solid line.

\section{$3 \quad$ Results}

\section{Shear Layer and Wake Modes}

The two-dimensional computations with laminar upstream boundary layers reveal an transition between two fundamentally different modes of cavity oscillations. These modes are termed, following Gharib and Roshko, ${ }^{26}$ as shear layer mode and wake mode. The runs performed show that transition from shear layer to wake mode occurs as the length of the cavity is increased relative to the upstream boundary layer thickness (for constant $L / \theta$ and $\operatorname{Re}_{\theta}$ ), and as $M$ is increased with other parameters constant.

The shear layer mode is characterized by the rollup of vorticity in the shear layer and impingement on the downstream cavity edge. The frequencies are in reasonable agreement with those predicted by the Rossiter equation, and consist primarily of Rossiter modes 1 and 2. ${ }^{8}$ Spectra and frequencies of oscillation are discussed in more detail below. Qualitatively, the iso-contours of vorticity depicted in figure 3 (for run L2) are representative of the shear layer mode of oscillation. The cavity is relatively quiescent, with a weak vortex occupying the latter half of the cavity. Vorticity of the opposite sign (to boundary layer vorticity) is generated along the walls of the cavity. Note that at two different instants in time, while the phase of the disturbances 
in the shear layer has shifted, the vorticity contours in the cavity are nearly the same. Animations of the contours confirm that the interaction of the flow in the cavity with the shear layer is relatively weak.
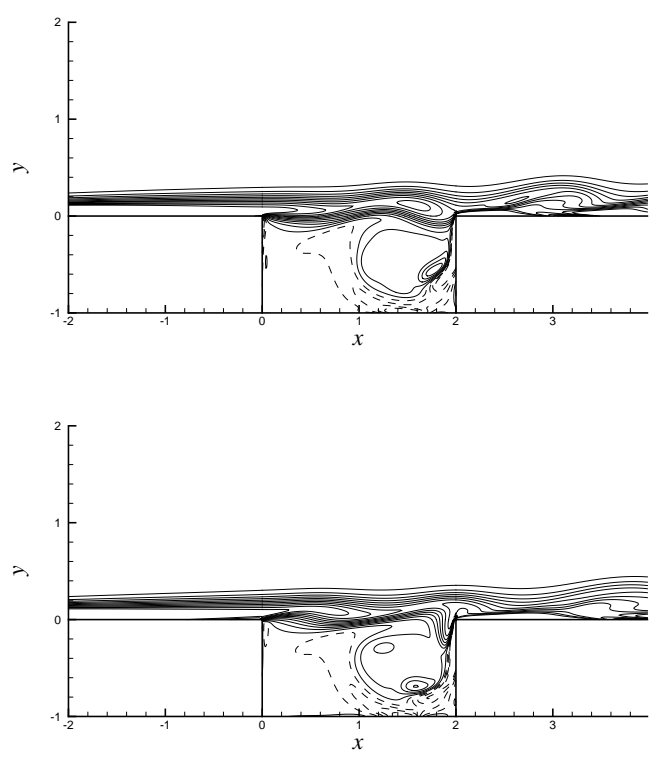

Figure 3: Instantaneous vorticity contours at two different instants during the oscillation cycle for run L2 (shear layer mode). 15 contours between $\frac{\omega D}{U}=$ -5 and 1.67. Positive contours are dashed. Only a small portion of the computational domain near the cavity is shown.

The wake mode is characterized by a large scale shedding from the cavity leading edge, in a manner similar to wake flows. The shed vortex has dimensions of nearly the cavity size, and as it is forming, boundary layer fluid is directed into the cavity. The vortex is shed from the leading edge and ejected from the cavity in a violent event. The vortex is large enough to cause flow separation upstream of the cavity during its formation, and again in the boundary layer downstream of the cavity as it convects away. We believe the upstream separation is a key feature of the transition to wake mode, and is discussed further below. Figure 4 shows two snapshots of the vorticity field in wake mode for run L4.

Time traces of the normal velocity at $y=0$ and $x=3.13 D$ are shown in figure 5 , for the series of runs L1-L5, where $L / \theta$ was varied, with constant $D / \theta$. It is evident that the transition from shear layer mode to wake mode occurs as $L / \theta$ is increased through 75. For $L / \theta=25$, the oscillations are damped and the flow becomes steady. For $L / \theta=75$, it appears
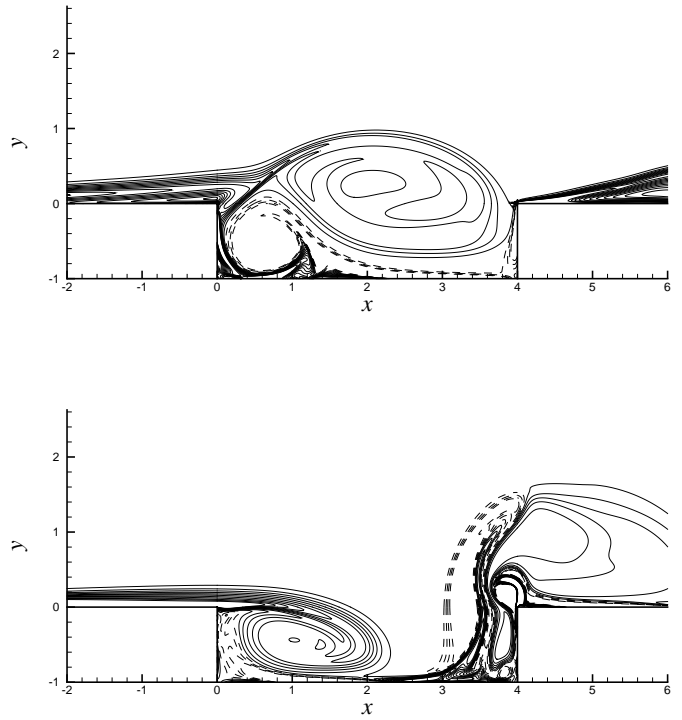

Figure 4: Instantaneous vorticity contours at two different times during the oscillation cycle for run L4 (wake mode). 15 contours between $\frac{\omega D}{U}=-5$ and 1.67. Positive contours are dashed. Only a small portion of the computational domain near the cavity is shown.

that there is mode switching, with wake and shear layer modes being present at different times. The transition is also a function of $M$, and for $L / \theta=102$, shear layer mode exists for $M<0.3$ and wake mode for $M>0.3$. Again, time traces for flows near the transition indicated the presence of mode switching.

Another important difference between the two modes is their radiated acoustic field. The instantaneous iso-contours of dilatation are plotted over the entire computational domain (except for the unphysical buffer region) in figure 6 for runs L4 and L2. The former is oscillating in wake mode while the latter is in shear layer mode. The acoustic fields are quite different. For shear layer mode, the acoustic field, centered at the downstream cavity edge, is dominated by a single frequency, corresponding to Rossiter mode 2. The most intense radiation occurs at an angle of approximately 145 degrees from the streamwise direction. The acoustic field is intense enough to display nonlinear steepening of the waves. The compressions are dark contours, the expansions are light contours. The acoustic field in wake mode is much more complex, and displays a wide range of frequencies. Again, there is intense upstream radiation from the cavity edge, but the wavelength is 


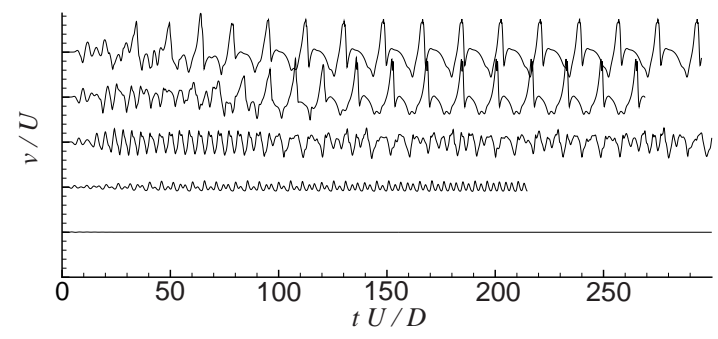

Figure 5: Time traces of the normal velocity, relative to $U$, at $y=0, x=3.13 D$ for the series of runs L1L5. The vertical axes have been artificially shifted to show all the data clearly, with major tick marks representing 1 unit.

longer by a factor of about 2, and the amplitude larger by a factor of about 4, compared to the shear layer mode. A very sharp acoustic pulse also emanates from the downstream edge. The origin of this wave is the ejection of the shed vortex from the cavity, depicted in figure 4 .

Gharib and Roshko ${ }^{26}$ performed experiments on incompressible cavities (an annular gap in an axisymmetric body in water), where the upstream boundary layer was also laminar. Transition between non-oscillatory, shear layer, and wake modes occurred at $L / \theta=80$ and $L / \theta=160$. Our data show that the change from wake mode to shear layer mode depends also on the Mach number. In table 1, we have indicated for each run the observed mode of oscillation. Note that for very short cavities, oscillations are damped and the flow eventually becomes steady, which was also observed in the experiments. As in the experiments, the drag is significantly higher in wake mode. The drag for several runs with different $L / \theta$ is given in table 1 .

\subsection{Spectra}

The spectra of the oscillations are substantially different in wake and shear layer mode. Figure 7 shows the spectra for a $L / D=2$ cavity with $M=0.7$ (run 2M7), which is oscillating in shear layer mode. In figure 8 , distinct peaks in the spectra are compared to predictions from the modified Rossiter formula:

$$
S t=\frac{f L}{U}=\frac{m-\alpha}{M \sqrt{1+\frac{\gamma-1}{2} M^{2}}+\frac{1}{\kappa}},
$$

where $m=1,2,3, \cdots$. The values $\alpha=0.25$ and $\kappa=0.57$ were used. ${ }^{9}$ Plotted in the figure are the series of runs $(2 \mathrm{M})$ with $L / D=2$ and $L / \theta=52.8$. The agreement is relatively good and within the scatter of experimental data $\left(e . g .^{11}\right)$. It needs also to be
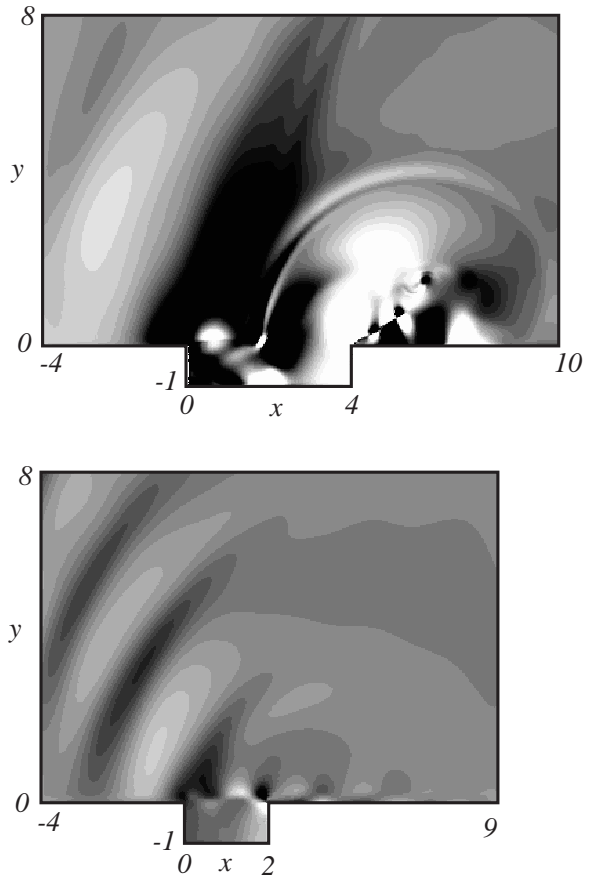

Figure 6: Acoustic field from the DNS. Top: Run L4 . Bottom: Run L2. Contours of dilatation are plotted. The entire domain except the buffer region near the boundaries is shown.

noted that the spectra were computed from computational data over relatively low numbers of periods (compared to say, experiments). Short time series, with necessary windowing techniques, can lead to significant leakage among the low frequencies. Nevertheless, the figure provides evidence that the basic instability mechanism in shear layer mode is the one envisioned by Rossiter.

The spectra in wake mode are very different. After an initial transient, which at early times is similar to shear layer mode, the flow becomes nearly periodic in time, with the fundamental period corresponding to the vortex shedding from the leading edge. A typical time series and its spectrum (for run L4) are shown in figure 9. After a transient, the fundamental mode oscillates at a Strouhal number of 0.25 , which is lower than Rossiter mode one (0.31), and additional peaks in the spectrum are all harmonics of the fundamental. Note that the initial transient was not used in computing the spectrum. In addition, there is very little variation of the fundamental frequency with $M$. For $M=0.4$ to $M=0.8$ the fundamental frequency varied less than $4 \%$, compared 


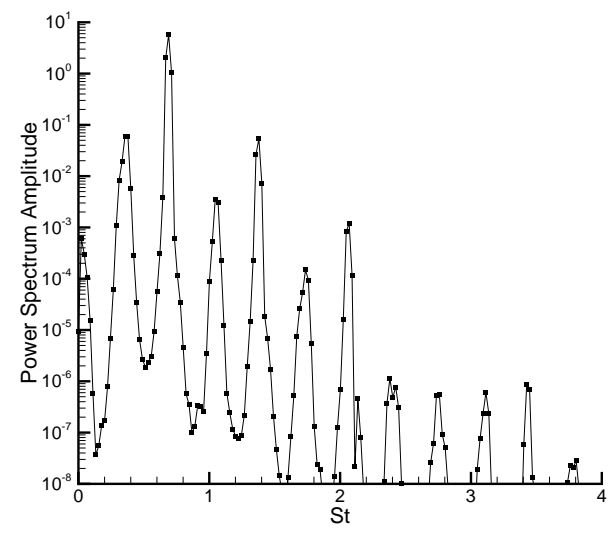

Figure 7: Spectrum of normal velocity at $y=0$ and $x=1.57 D$, for run $2 \mathrm{M} 7$.

to the expected variation of about $20 \%$ for Rossiter mode one. The $4 \%$ variation is, in fact, within the uncertainty in frequency associated with the total sampling period. The lack of variation with $M$ indicates that the mode is not acoustically driven, and it appears that the feedback in this case is provided by the complicated recirculating flow in the cavity.

\subsection{Wake mode and unsteady laminar separation}

It should be noted that the transition from shear layer to wake mode, detected in incompressible experiments and the present compressible computations, appears not to have been noted in previous compressible experiments. The very low Reynolds number of the calculations, and the laminar state of the upstream boundary layer could be the cause of wake mode. For the computations, $\mathrm{R} e_{\theta}$ is on the order of 100 , which is of the same order as in the incompressible experiments, ${ }^{26}$ but much lower than any compressible experiments. While it is unlikely that the shear layer dynamics are highly dependent on Reynolds number, even for $\operatorname{Re}_{\theta}$ as low as 100, the impact of the oscillations on an upstream laminar boundary layer could be very different than for a turbulent one.

The computations show that in wake mode, the boundary layer alternately separates and reattaches well upstream of the cavity edge, due to the oscillating adverse pressure gradient caused by the vortex shedding. In fact, the computational results suggest that laminar boundary layer separation upstream of the cavity is the cause of the transition to wake mode.

For steady separation, Thwaites method provides

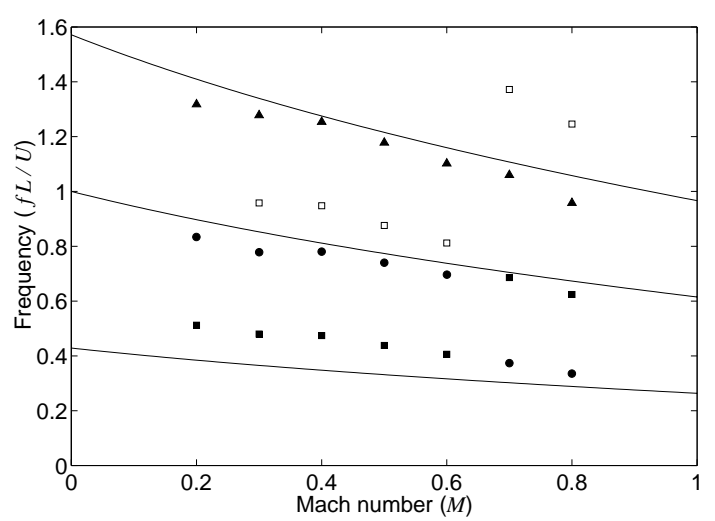

Figure 8: Strouhal numbers for peaks in spectra for the $2 \mathrm{M}$ series of runs (shear layer mode). The square symbols indicate the highest amplitude mode, circles the second highest, and triangles, the third highest. The open squares are peaks in the spectra at the first harmonic of the highest amplitude Rossiter mode. The lines are equation (1) with $m=1,2$ and 3 .

a simple way to estimate the magnitude of an adverse pressure gradient needed to cause separation $\left(e . g .{ }^{27}\right)$. The criterion is:

$$
-\lambda=\frac{\theta^{2}}{U \nu} \frac{d P}{d x}=0.09 .
$$

For unsteady and oscillatory separation, this simple criterion is not expected to hold. However, the Rossiter modes, and thus the oscillating pressure gradient that the boundary layer sees, have fairly low frequency compared to appropriate boundary layer time scales, and it might be expected that the separation criterion could be considered quasi-static.

Thus we use equation (2) as a criterion for separation, and, hence, transition to wake mode. We estimate the value of $\lambda$ at the cavity leading edge as a function of time, starting from the initial condition, for six different runs in figure 10. The middle frame corresponds to two different runs in wake mode. It is apparent that the oscillations, starting at zero, are initially in shear layer mode, and that transition occurs only after a substantial period of growth (in time) of the modes. The transition to wake mode occurs around the time when $-\lambda$ exceeds 0.09. For the runs that remain in shear layer mode (bottom frame), $-\lambda$ saturates around -0.02 . Finally, the top frame, corresponds to a run (L3) that exhibited mode switching, and $-\lambda$ reaching as high as 0.07. Thus the simple laminar separation criterion appears to predict the onset of wake mode surprisingly well. 

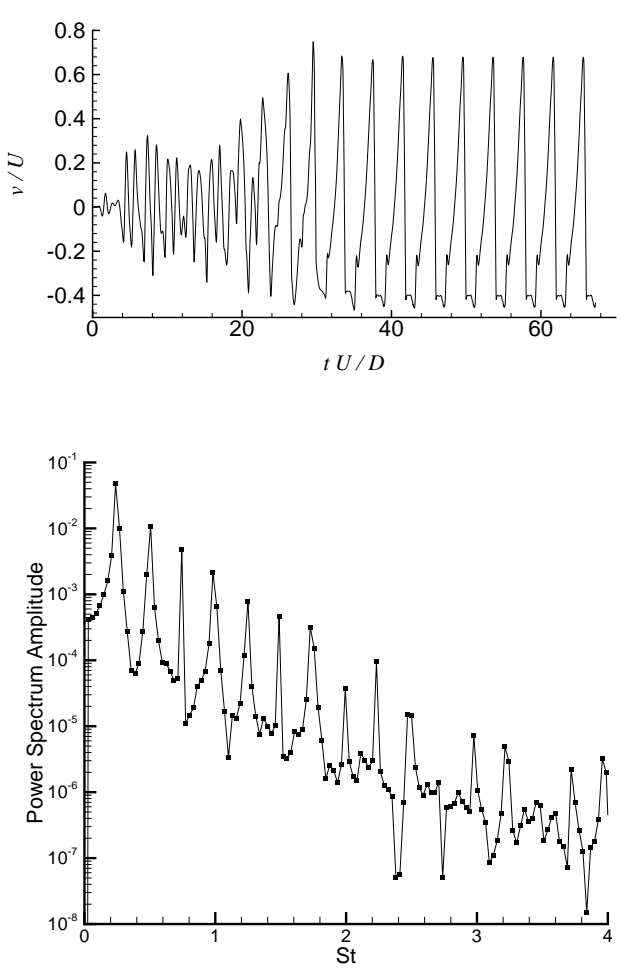

Figure 9: Run L4. Top: normal velocity versus time at $y=0$ and $x=3.13 D$. Bottom: spectrum of the normal velocity at $y=0$ and $x=3.13 D$.

The estimates for $\lambda$ were based on the initial shear layer thickness at the cavity edge, and the timedependent pressure fluctuations at the leading edge, and we are currently exploring more refined computations of the upstream boundary layer characteristics.

At present, we have detected the transition, holding $D / \theta$ constant and increasing $L / D$ (and therefore also $L / \theta$ ) and increasing $M$ holding other parameters constant. This result suggests that in order to be relevant to typical compressible cavity experiments, numerical simulations with laminar upstream boundary layers should avoid parameter regimes where wake mode can be expected. It is not clear, at present, what role $L / \theta$ plays in the transition, although a previous study by Sarohia, ${ }^{28}$ which had a laminar boundary layer upstream, suggested that the shear-layer mode instability characteristics were independent of $D / \theta$. Further numerical experiments are planned to explore different trajectories through parameter space to further characterize the transition.

A turbulent boundary layer would be much more resistant to such separation and may preclude the
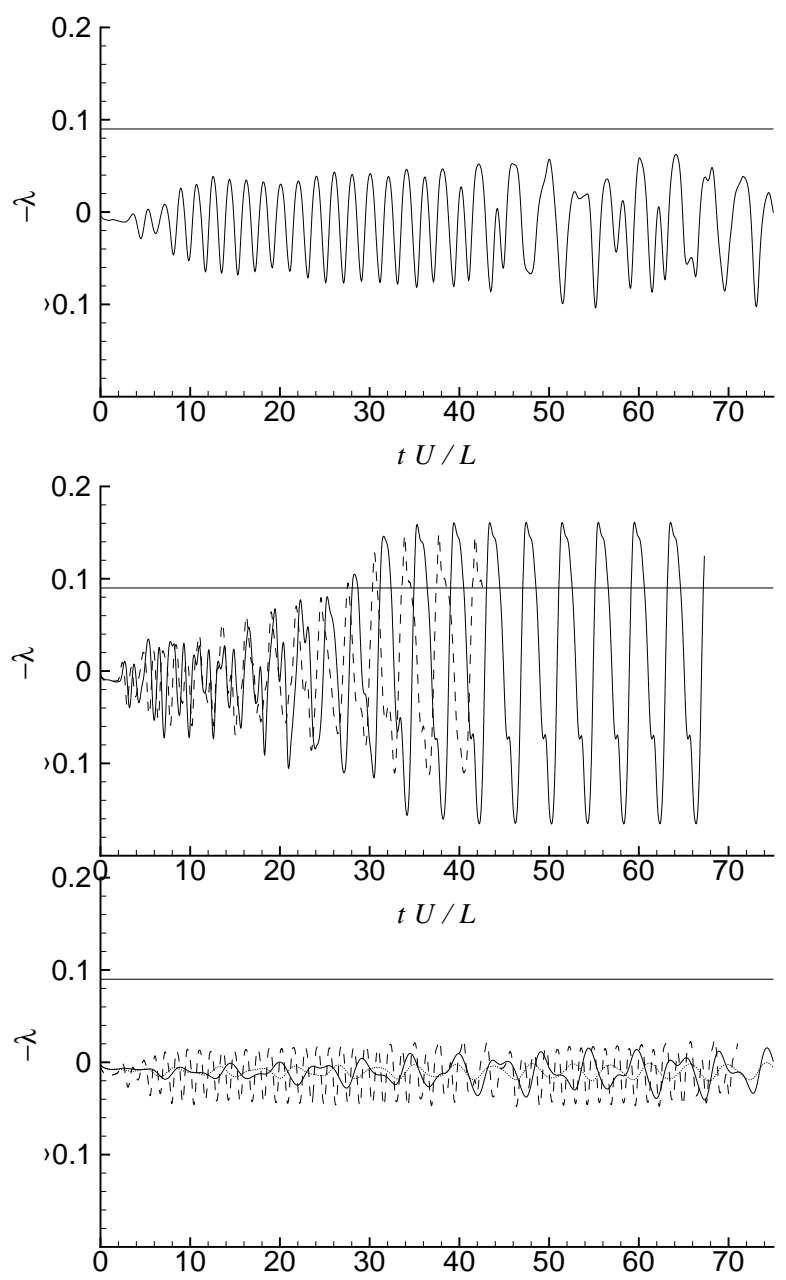

Figure 10: Estimated Thtwaites parameter, $\lambda$, at the upstream cavity edge. Top: mixed mode (L3). Middle: wake mode (L4 $\longrightarrow$, 4M4 ----). Bottom: shear layer mode (L2 —, R1 ---, 2M4 …....). The solid line indicates the approximate separation criterion.

emergence of wake mode. We speculate that this is the reason why wake mode has not been detected in previous compressible cavity experiments. Of course, it is impossible to rule out that other Reynolds number and three-dimensional effects act to preclude the wake mode. Further investigations are needed to fully resolve the issue.

\section{$4 \quad$ Summary}

The results of the numerical simulations show that, for shorter cavities, compared to the upstream boundary layer thickness, and lower Mach numbers, the cavity oscillates in a shear layer mode, which is consistent with the shear layer instability/acoustic 
feedback mechanism of Rossiter. ${ }^{8}$ The spectra show peaks corresponding to Rossiter modes 1, 2, and 3 . Acoustic radiation is intense and directional, but dominated by a single frequency corresponding to mode 2. There is little interaction between the shear layer, and a weak vortex that occupies the downstream half of the cavity. For longer cavities, and higher Mach numbers, the cavity oscillations become nearly periodic in time, with one cycle corresponding to the growth, shedding, and ejection of a very large vortex. In this wake mode the Strouhal number of the oscillations is nearly independent of Mach number. During growth, the boundary layer fluid is directed into the cavity and the cavity drag is very large. Ejection is accompanied by a sharp acoustic pulse. The vortex is strong enough to cause boundary layer separation both upstream of the cavity, during formation, and downstream of the cavity, after ejection. A similar transition was noted in the incompressible experiments of Gharib and Roshko ${ }^{26}$ (who also had a laminar upstream boundary layer), but has not been seen in compressible experiments at higher Reynolds numbers. The results suggest that the laminar separation upstream of the cavity is, in fact, responsible for the transition to wake mode. We speculate that when the upstream boundary layer is turbulent, the increased resistance to separation may prevent wake mode oscillations.

\section{Acknowledgements}

This research was supported by AFOSR under grant F49620-98-1-0095 with technical monitor Dr. Thomas Beutner. Supercomputer time was provided by the Department of Defense High Performance Computing centers, as well as the National Science Foundation. C.W. Rowley acknowledges the support of a National Science Foundation Graduate Fellowship.

\section{References}

[1] M. Gharib. Response of the cavity shear layer oscillations to external forcing. AIAA J., 25(1):43-47, 1987.

[2] A. D. Vakili and C. Gauther. Control of cavity flow by upstream mass injection. AIAA Paper 91-1645, 1991.

[3] A. M. Lamp and N. Chokani. Active control of compressible cavity flows using a small jet. AIAA Paper 96-0446, 1996.

[4] L. Shaw. Active control for cavity acoustics. AIAA Paper 98-2347, 1998.
[5] L. N. III Cattafesta, S. Garg, M. Choudhari, and F. Li. Active control of flow-induced cavity resonance. AIAA Paper 97-1804, 1997.

[6] L. Mongeau, H. Kook, and M. A. Franchek. Active control of flow-induced cavity resonance. AIAA/CEAS Paper 98-2349, 1998.

[7] T. Kestens and F. Nicoud. Active control of an unsteady flow over a rectangular cavity. AIAA Paper 98-2348, 1998.

[8] J. E. Rossiter. Wind-tunnel experiments on the flow over rectangular cavities at subsonic and transonic speeds. Technical Report 3438, Aeronautical Research Council Reports and Memoranda, October 1964.

[9] C. K. W. Tam and P. J. W. Block. On the tones and pressure oscillations induced by flow over rectangular cavities. J. Fluid Mech., 89:373399, 1978.

[10] A. B. Cain, W. W. Bower, F. McCotter, and W. W. Romer. Modeling and prediction of weapons bay acoustic amplitude and frequency. Technical report, VEDA Inc., 1996.

[11] L. N. III Cattafesta, M. S. Kegerise, and G. S. Jones. Experiments on compressible flowinduced cavity oscillations. AIAA Paper 982912, 1998.

[12] D. Fabris and D.R. Williams. Experimental measurements of cavity and shear layer response to unsteady bleed forcing. AIAA Paper 99-0605, 1999.

[13] X. Zhang, A. Rona, and J. A. Edwards. The effect of trailing edge geometry on cavity flow oscillation driven by a supersonic shear layer. The Aeronautical Journal, pages 129-136, March 1998.

[14] D. F. Fuglsang and A. B. Cain. Evaluation of shear layer cavity resonance mechanisms by numerical simulation. AIAA Paper 92-0555, 1992.

[15] T. Colonius, S. K. Lele, and P. Moin. Sound generation in a mixing layer. J. Fluid Mech., 330:375-409, 1997.

[16] T. Colonius, S. K. Lele, and P. Moin. The scattering of sound waves by a vortex-numerical simulations and analytical solutions. J. Fluid Mech., 260:271-298, 1994. 
[17] B. E. Mitchell, S. K. Lele, and P. Moin. Direct computation of Mach wave radiation in an axisymmetric supersonic jet. AIAA J., 35(10):1574-1580, October 1997.

[18] J. B. Freund, S. K. Lele, and P. Moin. Direct simulation of a Mach 1.92 jet and its sound field. AIAA paper 98-2291, 1998.

[19] Sanjiva K. Lele. Compact finite difference schemes with spectral-like resolution. J. Comput. Phys., 103(1):16-42, November 1992.

[20] C. W. Rowley and T. Colonius. Discretely nonreflecting boundary conditions for linear hyperbolic systems. submitted to J. Comput. Phys., 1999.

[21] T. Colonius, S.K. Lele, and P. Moin. Boundary conditions for direct computation of aerodynamic sound. AIAA J., 31:1574-1582, 1993.

[22] J. B. Freund. Proposed inflow/outflow boundary condition for direct computation of aerodynamic sound. AIAA J., 35(4):740-742, April 1997.

[23] Kevin W. Thompson. Time dependent boundary conditions for hyperbolic systems. J. Comput. Phys., 68:1-24, 1987.

[24] T. Poinsot and S. K. Lele. Boundary conditions for direct simulation of compressible viscous flows. J. Comput. Phys., 101:104-129, 1992.

[25] T. Colonius, S. K. Lele, and P. Moin. Boundary conditions for direct computation of aerodynamic sound generation. AIAA J., 31(9):15741582, September 1993.

[26] M. Gharib and A. Roshko. The effect of flow oscillations on cavity drag. J. Fluid Mech., 177:501-530, 1987.

[27] F. M. White. Viscous Fluid Flow. McGraw Hill, second edition, 1991.

[28] V. Sarohia. Experimental and analytical investigation of oscillations in flows over cavities. AIAA J., 15:984-991, 1977. 